\title{
BIM-Based Sustainability Certification of Building Components in the Construction Phase
}

\author{
Re Cecconi Fulvio, Moretti Nicola, Mannino Antonino and Dejaco Mario Claudio \\ Department of Architecture, Built Environment and Construction Engineering, Politecnico di Milano, via G. Ponzio 31, Milano \\ 20133, Italy
}

\begin{abstract}
Building Information Modelling (BIM) is becoming increasingly present in every stage of assets' lifecycle. More and more, the BIM approach is related to the sustainability assessment protocols which play an important role in reducing the impact on the environment. In the design and construction phase, the products' sustainability certification is a key issue to be managed and controlled, to achieve higher efficiency in operations. Through this research, a BIM-based methodology to automate the sustainability certification process in construction phase has been developed. According to the proposed method, the contractor proposes a building component to the work supervisor, by uploading the related technical datasheet in a Common Data Environment (CDE). If the component meets the performance requirements defined for the construction stage and agreed with the involved parties (work supervisor, client and sustainability accredited professional), it is validated and uploaded in the BIM model by the BIM manager. The methodology has been tested in a case study, confirming the effectiveness of the proposed approach. However, it should be further validated. Moreover, it can be improved and a higher level of automation can be achieved, in order to cope with product dictionaries and templates under development in CEN technical committee 442.
\end{abstract}

Key words: BIM, leadership in energy and environmental design (LEED), sustainability certification, green BIM.

\section{Introduction}

In the last few decades, sustainability, has become one of the key subjects in the Architecture, Engineering, Construction and Operation (AECO), under the general aim of reducing the impact of the construction process on the environment [1]. The AECO sector is responsible both for the consumption of a great amount of resources (e.g. water, materials, energy) and the production of waste and harmful atmospheric emissions [2, 3]. Therefore, it is necessary to better manage both the consumption of resources and the energy usage. According to the Directive 2010/31/EU [4], in the European Union buildings account for $40 \%$ of total energy consumption and $35 \%$ of the total amount of greenhouse gas emissions. The need to reduce the impact of buildings on the environment, also thanks to

Corresponding author: Mannino Antonino, Ph.D. student, research fields: building technology and construction management. sustainable design choices, led to the development of green building standards, certifications and sustainability rating systems [5]. These rating systems and certification are based on life-cycle parameters, such as: planning and design innovation, site sustainability, energy efficiency, water efficiency and management, indoor quality, material/resources efficiency and management.

Although most of the parameters used in the sustainability assessments are quite similar (e.g. energy consumption, water efficiency, building location), there are many different types of certification. The most acknowledged sustainability rating systems are [6]:

- Building Research Establishment Environmental Assessment Method (BREEAM);

- Leadership in Energy and Environmental Design (LEED);

- Sustainable Building Tool (SBTool).

LEED and BREAM can be included among the 
most used and robust rating systems on the international scene, although it is possible that in some cases parameters that describe the same performance are calculated differently [7]. In order to promote an uniformity in the sustainability assessment of the built environment, some efforts to harmonize the indicators have been carried out by the CESBA protocol [7]. However, complexity of these rating system has increased more and more over the past few years, also due to an increase in user and clients requests for different versions of the rating system, in order to analyze different aspects of the built environment (e.g. new construction, existing buildings, operation, site, core \& shell) [8]. Despite this complexity, these rating systems can be considered, and also more and more used, to justify an increase of the building market value rather than for the effective achievement of sustainability objectives [9].

Moreover, several building products certifications are also recognized within buildings sustainability rating systems (e.g. LEED, Green Globes and the National Green Building Standard) as a result of growing concerns about products toxicity and their impact on indoor environmental pollution.

In this context, despite some attempts to provide a semi-automated process in the sustainability certifications of buildings during the design phase can be found in Refs. [6, 9-12], fewer studies have been carried out aiming at automating the certification process during the construction phase. This article proposes a methodology to semi-automate the process of LEED sustainability certification of buildings during the construction stage (stage number 5 in the RIBA Plan of Work [13]). This methodology is based on the information management workflow proposed by the ISO 19650 part 1 and 2 [14]. Finally, the methodology has been validated through the application on a case study, an office building in Italy, showing good preliminary results both in time savings and in the improvement of several authorization steps during the whole process.

\section{State of the Art}

LEED protocol was introduced in 1993 aiming at minimizing environmental impacts of built environment starting from design phase up to its use and decommissioning, through energy/resources efficiency actions [15]. LEED plays a significant role among green building certification systems [16-21] and most of the green building projects are based on it [22]. The computation of the LEED sustainability ranking of an office building (BD $+\mathrm{C}$ : New Construction) is carried out through the use of a checklist which comprises credits about several sustainability categories: Location and Transportation (up to 16 points), Sustainable Sites (up to 10 points), Water Efficiency (up to 11 points), Energy and Atmosphere (up to 33 points), Materials and Resources (up to 13 points), Indoor Environmental Quality (up to 16 points), Innovation in Design (up to 6 points) and Regional Priority (up to 4 points) [23, 24]. The assessment of the project is based on a scoring system. The United States Green Building Council (USGBC) established four different levels for LEED certification: Certified, Silver, Gold and Platinum [23, 24]. The increased demand by clients and users, has led to the definition of different versions of protocols to assess different parts or life cycles of the building (e.g. new construction, existing buildings, operation, site, core \& shell) and of the neighborhood [7].

\subsection{Building Information Modeling and Sustainability Assessment}

Building Information Modelling (BIM) allows the digital modelling and information management of the physical asset, where different semantics can be related to the building components, group of components and spaces [1]. One of the most innovative application in this field is called Green BIM, an employment of BIM-based models and processes for the achievement of sustainability objectives (energy performance analysis, sustainable 
retrofit, etc.) [3]. Through the use of BIM methodology is possible to better manage buildings' data and information throughout the life cycle, and many efforts have been made in order to use this methodology to develop Green Buildings $[25,26]$ and their sustainability assessment [7]. Moreover, it is possible to improve interoperability exchanging information between BIM software and rating systems also relying on Machine View Definitions (MVD) of standard protocols such as Industry Foundation Classes (IFC) [27] or Construction Operations Building Information Exchange (COBie) [28]. These standards improve data and information exchange and management among parties throughout various project stages. Both COBie and IFC standards could act as a medium to improve sustainability assessment because they allow data exchange between different software applications during the whole lifecycle of the building [29]. To date, BIM methodology is not yet widely used for sustainability assessment during the construction phase. Therefore, an innovative BIM-based approach to facilitate the sustainability certification of building products during this life cycle phase has been developed. The proposed certification process is carried out through the procedures characterizing the Common Data Environment (CDE): the shared virtual environment in which virtual model and buildings data/information are managed. This virtual model, also known as Federated Model and in which models coming from different disciplines are integrated, could be considered also as the starting point for the development of the Digital Twin (DT) of the building [30]. Through the use of a CDE is possible to work in a truly shared environment in which communication quality among all the stakeholders of the process is improved and data are constantly updated. A CDE can assume four different states (Work in Progress, Shared, Published Documentation, Archive) according to the advancement in the production of the information managed [14]. ISO 19650-1:2018 [14] describes also the modalities allowing the exchange of information within different stakeholders. Accordingly, the development of the proposed BIM-based methodology for sustainability certification of building has been developed around these principles.

\section{Methods and Tools}

Sustainability certifications involve both the design and the construction phases. The process proposed in this research aims to improve the assessment of LEED protocol criteria in the construction phase which involves several stakeholders and, often, involves time-consuming procedures for the communication among them. During the construction stage a lot of data and information about construction products and systems are produced and shared among involved parties. Typical construction information includes an owner's set of documents transferred from the contractor at the end of the project in a number of files [31]. The BIM methodology and CDE rules can be used to improve data and information sharing during the construction stage, giving quick responses and avoiding ineffective communication which could generate issues in the building sites [32]. According to the process presented in Fig. 1, the BIM model is created in the design phase and developed with a suitable level of geometric and semantic detail for the construction site. With this premise, the process starts and proceeds according to the phases here defined:

(1) Building product proposal. The process starts with a building product proposal by the supplier. The proposal must meet the project requirements stored in the digital model of the building.

(2) Technical verification. Subsequently, there is a first review and authorization step by the work supervisor who has the task of assessing whether the product meets the project requirements or not.

(3) Elective client authorization. After the first product check, if passed, a further authorization step by the client could be required (e.g. in case of very 


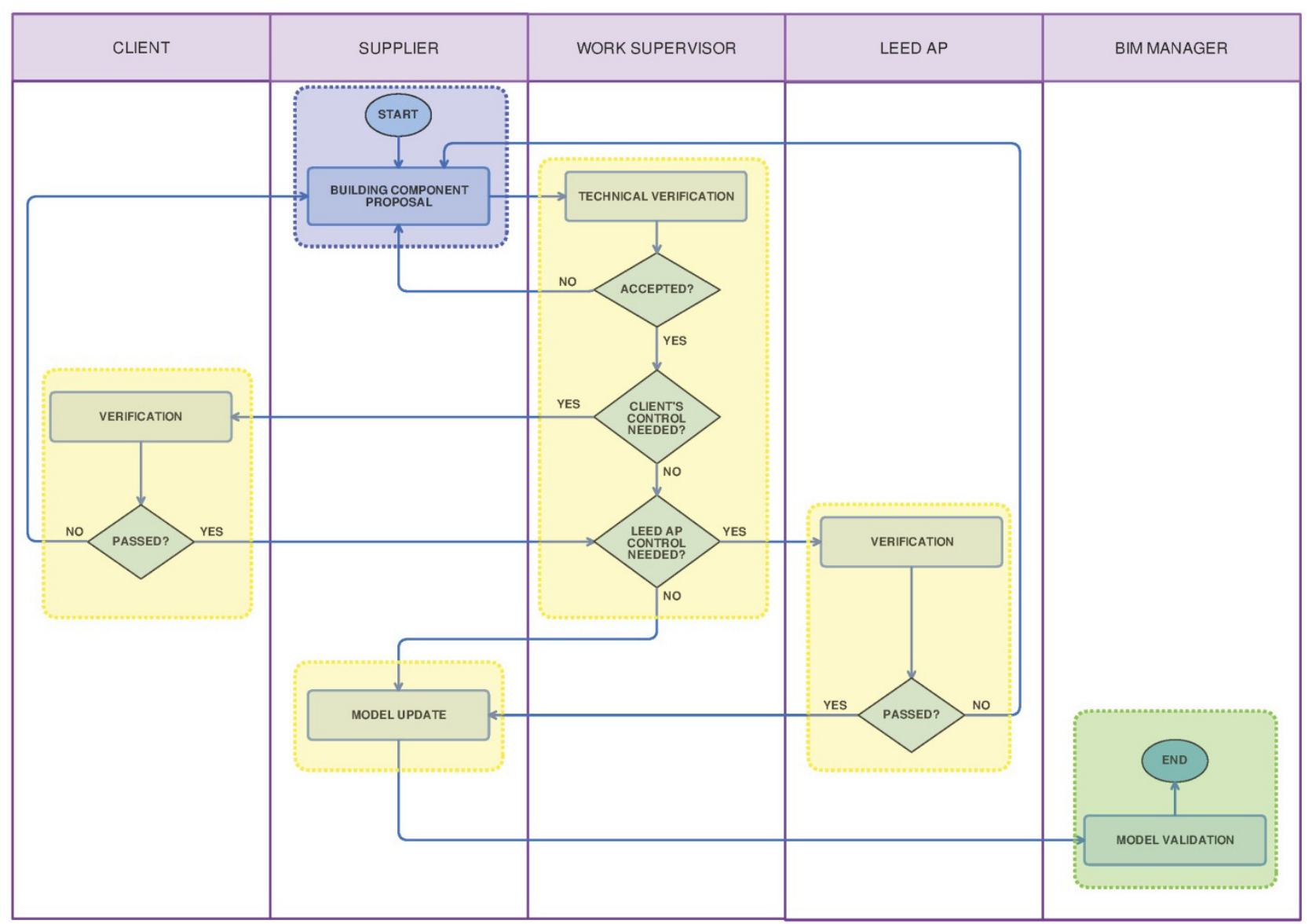

Fig. 1 BIM based LEED certification process. Color according to CDE stages: work in progress (blue), shared (yellow), and published (green).

critical equipment or expensive product). The work supervisor decides whether the client authorization is required or not.

(4) LEED accredited professional (AP) authorization. Once the previous authorizations have been obtained, the next phase concerns the verification of the building component by the LEED AP who checks the technical specifications of the proposed product and assigns a score according to the LEED's procedures.

(5) BIM manager check and validation. Finally, if all authorizations are obtained, the supplier must update the digital model. The BIM manager checks if the model has been updated correctly, without clashes and with all the required information about the building product. Finally, the BIM manager can validate the updated model.
As mentioned before, not all authorization steps are compulsory. It is possible that, for a certain types of building component, clients' authorizations are not needed. In other cases, components do not significantly affect the sustainability rate of the building and, in these cases, the process will be carried out without the LEED AP authorization step.

\section{Application of the Proposed Approach}

The proposed approach has been tested on a case study concerning the LEED certification of building components in an office building in Italy.

The building (Fig. 2) consists in a single block (20 $\mathrm{m} \times 93 \mathrm{~m}, 1,860 \mathrm{~m}^{2}$ typical floor area) with a glass curtain wall. It has three floors above the ground and underground parking. Two stairwells divide all the floors of the building in three parts. The structure is 


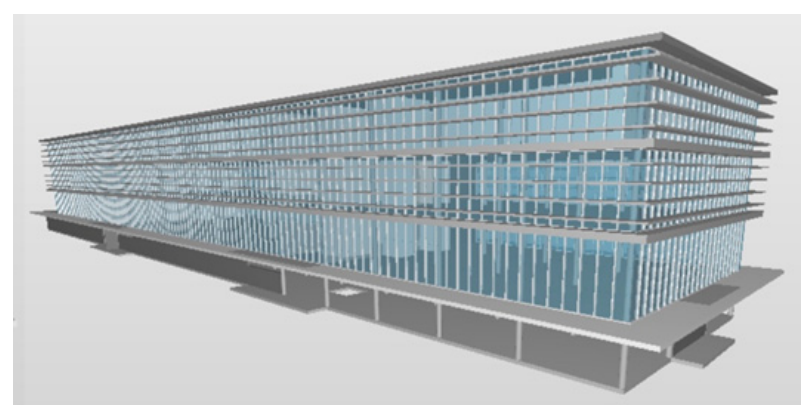

Fig. 2 North/east facade of the building (IFC model).

made by concrete pillars and beam. For the application of our methodology on the case study, the second-floor curtain wall panel has been considered.

The building has been modeled with Autodesk Revit 2018, and Autodesk BIM 360 DOCS has been employed as document management systems (DMS) to store relevant data and information and to optimize the sharing among the involved parties.

To improve interoperability, a DMS that allows visualizing and checking the digital model both in proprietary (.rvt) and IFC format has been used. In this way it is possible to extract information not only from digital documents uploaded by stakeholders, but also from virtual components within the federated model.

The DMS has been structured creating folders organized by building component and following the structure of the Work Breakdown Structure (WBS). For each building component folder, sub-folders organized by building floors have been created. Finally, once the DMS structure has been completed, data sheets of each building component have been uploaded in the appropriated folder (Fig. 3).

In this case, the proposed approach has involved all the five stakeholders of the process. In the DMS, according to the WBS, has been created a folder structure with property fields to allow an easy visualization of technical data and information about building components (e.g. thermal transmittance for windows).

In this case study, folders (Fig. 4) have been created manually by the BIM manager, who is also the administrator of the DMS. Once the DMS has been set up reproducing the WBS of the building, as first step of the process, the supplier can upload the data sheet of the building product on the DMS (in this case a window to be installed at second floor). Since the DMS allows visualizing assets both in the proprietary format and the IFC open format, the supplier could also upload the 3D model of the building component to be verified. Accordingly, it is possible to manage data and information in a more efficient way.

Moreover, the building component's attributes previously set by the BIM manager in the DMS folders, have been compiled by the supplier with data needed for LEED evaluation extracted from the data sheet. An example concerning the thermal transmittance has been employed for the calculation of the LEED score in the "Energy and Atmosphere Credit: Optimize Energy Performance is shown in Fig. 5.

This credit allows obtaining 18 points of the LEED protocol for office building, according to the achieved

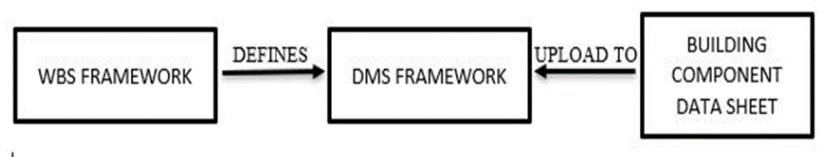

Fig. 3 Working on a DMS.

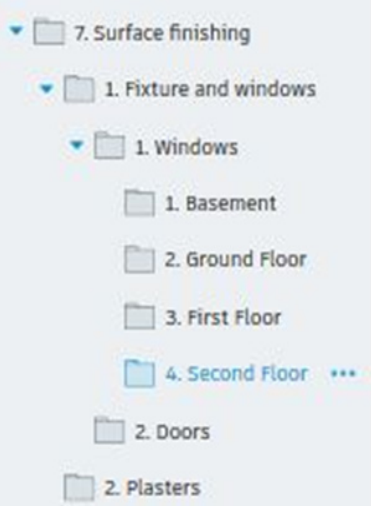

Fig. 4 DMS folders structure.

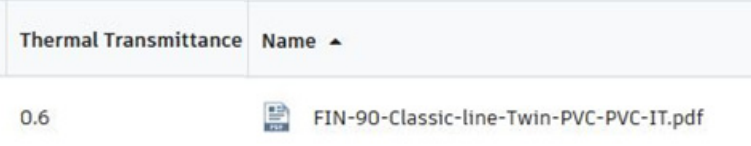

Fig. 5 Upload data sheet in the appropriate folder. 


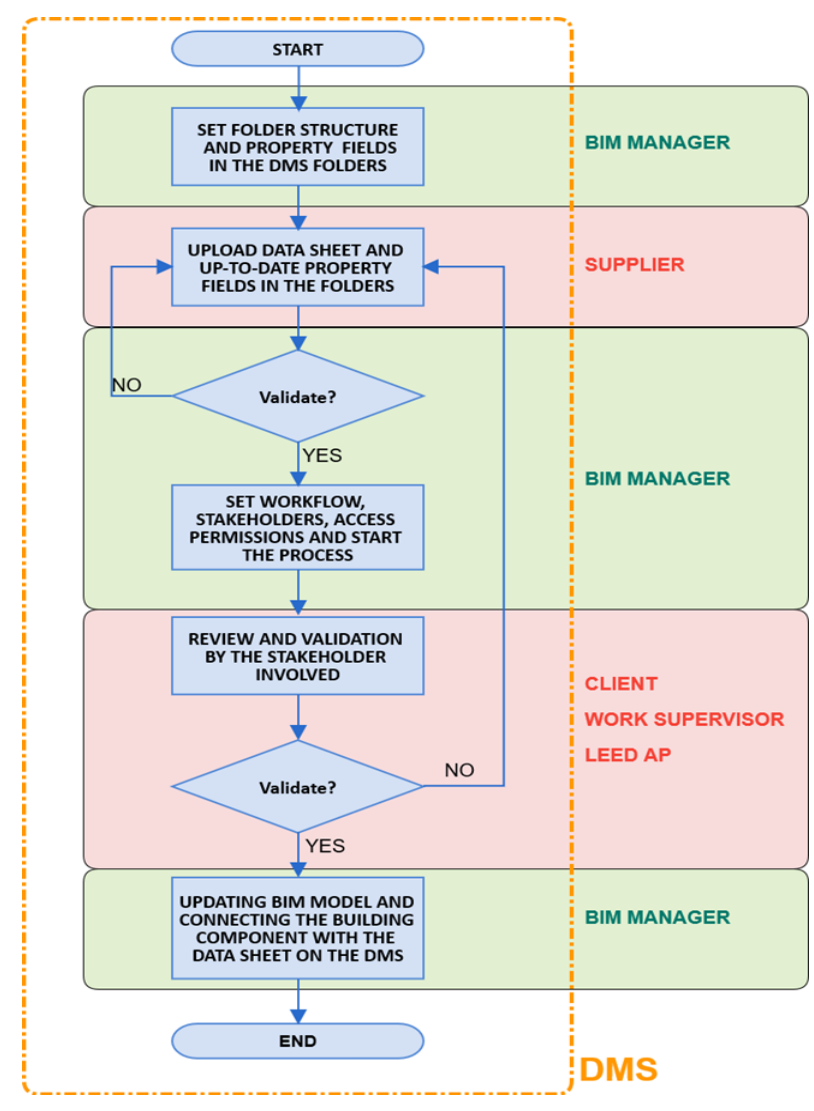

Fig. 6 Three step approval workflows.

Type Parameters
\begin{tabular}{|l|l|}
\hline \multicolumn{1}{|c|}{ Parameter } & \\
\hline Identity Data & B 2020100 \\
\hline Assembly Code & $0.5 \mathrm{~h}$ \\
\hline Fire Rating & \\
\hline Type Image & \\
\hline Keynote & Top 90 Step-line KAB \\
\hline Model & Finstral \\
\hline Manufacturer & \\
\hline Type Comments & http://127.0.0.1:8080/share/page/ \\
\hline URL & \\
\hline Description & \\
\hline Cost & Windows \\
\hline Assembly Description & 22 \\
\hline Type Mark & 23.30 .20 .17 .17 .14 \\
\hline OmniClass Number &
\end{tabular}

Fig. 7 DMS link in Revit.

improvement in energy performance of the new building.

The following steps concern the review and the authorization sequences of the building component specifications. To optimize this authorization process, a multi-approval steps workflow within the DMS has been created and it has been assigned to the building component. The workflow envisages the involvement of all the three stakeholders (Work Supervisor, Client and LEED AP) in the authorization process. The review of the building component is carried out by verifying the values extracted from the technical sheets and stored in the DMS folders. From the analysis of these values, each stakeholder decides whether to approve a component or not.

In this case study the work supervisor approved the building component, and the review task has been forwarded to the Client, which through the same procedure followed by the work supervisor but considering different parameters (e.g. costs), approved the component and forwarded it to the last stakeholder of the process: the LEED AP. In this last authorization-step, it has been verified that the building component meets LEED requirements through product properties retrieved from the data sheet stored by the supplier in the DMS (Fig. 6).

If one of these stakeholders does not approve the component, the process starts all over again.

Once also the LEED AP gives his approval, the building component has passed all the approval steps, and the supplier can update the digital (BIM) model with a final validation from the BIM manager who detects clashes and checks that the model has been updated correctly with the new data and information.

The connection between the digital building component within the BIM model and its technical data sheet within the DMS is carried out, in the BIM authoring software, through a link in the URL field within the "type properties" (Fig. 7).

\section{Results and Discussion}

The proposed Green BIM approach optimizes a process which is, to date, carried out in a fragmented way and without a predefined scheme. The strength of this process is the common shared environment in which the process is managed, without information loss and using a semi-automated certification process: once stakeholders and workflows are defined and 
assigned to the building components, the approval by each party automatically follows the workflow represented in Figs. 1 and 6.

However, in this paper the final LEED rating of the whole building has not been calculated. Nevertheless, the possibility to partially automate the computation of the sustainability ranking through the IFC protocol has been investigated [33], and the proposed methodology can be considered as a further step toward the automation in buildings sustainability certification within the context of the Green BIM.

Moreover, the possibility within the DMS environment not only to visualize and gather information from IFC open format but also from the proprietary format of the BIM authoring software, could improve both data visualization/organization and process information management. Furthermore, an efficient notification system and a cloud-based work could avoid errors and reduce time.

A weakness of the proposed process is that it still includes manual steps: for each building product, the BIM manager needs to create a blank field in the folders within the DMS. This field will be manually filled with products' information by the supplier, and this requires further effort in terms of time. Moreover, this manual procedure could be subject to errors that may be avoided in an automated process. Hence, further developments to improve automation in order to reduce errors and approval times for building components are required.

Finally, the approach proposed in this paper is related to the construction of a new building. In case of an existing building lacking a digital model, where its development requires much effort in terms of time and costs, COBie standard could be used. COBie is provided in the form of a spreadsheet and it is both human and machine readable. Once the COBie spreadsheet has been filled with the building data, it is possible to proceed with the sustainability assessment according to the process in Fig. 1. Strength is that a COBie spreadsheet is easy to handle and understand even for non-BIM-skilled professionals, it is compliant with several standards and can be used to implement a detailed digital model developed in subsequent phases. A weakness of using COBie in Green BIM is that many crucial data are missing in the COBie standards and thus have to be created as "Requirable" or "Additional" losing the interoperability across different platform.

\section{Conclusions}

The process presented concerns a semi-automated sustainability assessment through the integration of BIM methodology, within the information management workflow proposed by the ISO 19650 series (2018). Despite this approach is still at an early stage, the case study demonstrated how it was possible to carry out a semi-automated sustainability assessment of building products in the construction phase. A well-developed digital federated model is the starting point of this process. The federated model of the building includes all data and information about the different disciplines (structures, architecture and systems) and allows working in a truly shared environment. Moreover, the federated model can be considered as the starting point for the development of the DT of the building and will allow even greater optimization in the exchange and management of data and information. The BIM-based methodology for sustainability certification of building presented in this research has been developed following information exchange processes described in the ISO 19650-1:2018. Involved stakeholders can visualize data needed for building components approval or rejection. Once the building component is approved by all the stakeholders, the BIM model is updated and can be delivered as an as-built of the physical asset. The application of this process to the case study showed good preliminary results in saving precious time and resources, even if an improvement both of procedure and automation could further reduce errors and times. 
The implementation of the methodology provides a further step in the investigation of the capabilities offered by the Green BIM approach: the integrated use of BIM-based processes for the achievement of sustainability objectives. Next developments of the research concern the mapping of the LEED criteria according to the phase of the lifecycle of the asset and an investigation on how this approach could further improve the sustainability rating process in the design and construction stages.

To conclude, despite this methodology should be validated in further case studies and the automation of the process could be further improved, it proved to be a valid tool and may be implemented also in advanced version of the digital model (Digital Twin) of the built environment.

\section{References}

[1] Sanhudo, L. P. N., and Martins, J. P. D. S. P. 2018. "Building Information Modelling for an Automated Building Sustainability Assessment." Civ. Eng. Environ. Syst. 35 (1-4): 99-116.

[2] Eberhardt, L. C. M., Birgisdóttir, H., and Birkved, M. 2019. "Life Cycle Assessment of a Danish Office Building Designed for Disassembly.” Build. Res. Inf. 47 (6): 666-80.

[3] Wong, J. K. W., and Zhou, J. 2015. "Enhancing Environmental Sustainability over Building Life Cycles through Green BIM: A Review." Autom. Constr. 57: 156-65.

[4] European Parliament and European Council. 2010. "Directive 2010/31/EU of the European Parliament and of the Council of 19 May 2010 on the Energy Performance of Buildings (Recast)." Off. J. Eur. Union, 13-35.

[5] Vierra, S. 2016. "Green Building Standards and Certification Systems.” Building.

[6] Maltese, S., Moretti, N., Re Cecconi, F., Ciribini, A. L. C., and Kamara, J. M. 2017. "A Lean Approach to Enable Sustainability in the Built Environment through BIM." Techne-Journal Technol. Archit. Environ. 13: 278-86.

[7] Maltese, S., Tagliabue, L. C., Cecconi, F. R., Pasini, D., Manfren, M., and Ciribini, A. L. C. 2016. "Sustainability Assessment through Green BIM for Environmental, Social and Economic Efficiency." Procedia Eng. 180: 520-30.

[8] Y1ldiz, S. 2016. "Neighborhood Sustainability Assessment Tools and a Comparative Analysis of Five
Different Assessment Tools." J. Plan. 26 (2): 93-100.

[9] Wangel, J., Wallhagen, M., Malmqvist, T., and Finnveden, G. 2016. "Certification Systems for Sustainable Neighbourhoods: What Do They Really Certify?" Environ. Impact Assess. Rev. 56: 200-13.

[10] Soubra, S., Huovila, P., Scientist, P., Scientist, S., Palos, S., and Fies, B. 2012. "Linking IFCs and BIM to Sustainability Assessment." In Proc. CIM W78 2012 29th Int. Conf. Lebanon, 17-9.

[11] Jalaei, F., and Jrade, A. 2015. "Integrating Building Information Modeling (BIM) and LEED System at the Conceptual Design Stage of Sustainable Buildings." Sustain. Cities Soc. 18: 95-107.

[12] Ilhan, B., and Yaman, H. 2016. "Green Building Assessment Tool (GBAT) for Integrated BIM-Based Design Decisions." Autom. Constr. 70: 26-37.

[13] RIBA. 2013. RIBA Plan of Work 2013: Overview. London.

[14] ISO. 2018. EN ISO 19650-1:2018. Organization and Digitization of Information about Buildings and Civil Engineering Works, Including Building Information Modelling (BIM)-Information Management Using Building Information Modelling. Part 1: Concepts and Principles.

[15] Pham, D. H., Lee, J., and Ahn, Y. 2019. "Implementing LEED v4 BD + C Projects in Vietnam: Contributions and Challenges for General Contractor." Sustainability 11 (19): 5449.

[16] Mangialardo, A., and Micelli, E. 2019. "Does Sustainability Affect Real Estate Market Values? Empirical Evidence from the Office Buildings Market in Milan (Italy)." Sustainability 11 (1): 1-14.

[17] Amiri, A., Ottelin, J., and Sorvari, J. 2019. "Are LEED-Certified Buildings Energy-Efficient in Practice?" Sustainability 11 (6): 1-14.

[18] Uğur, L. O., and Leblebici, N. 2018. “An Examination of the LEED Green Building Certification System in Terms of Construction Costs." Renew. Sustain. Energy Rev. 81 (Mar.): 1476-83.

[19] Jeong, J., Hong, T., Ji, C., Kim, J., Lee, M., and Jeong, K. 2016. "Development of an Evaluation Process for Green and Non-green Buildings Focused on Energy Performance of G-SEED and LEED.” Build. Environ. 105: 172-84.

[20] Zhao, J., Lam, K. P., Biswas, T., and Wang, H. 2015. "An Online Platform to Automate LEED Energy Performance Evaluation and Submission Process." Constr. Innov. 15 (3): 313-32.

[21] Wu, P., Song, Y., Shou, W., Chi, H., Chong, H. Y., and Sutrisna, M. 2017. "A Comprehensive Analysis of the Credits Obtained by LEED 2009 Certified Green Buildings." Renew. Sustain. Energy Rev. 68 (April): 370-9. 
[22] Xia, B., Chen, Q., Xu, Y., Li, M., and Jin, X. 2015. "Design-Build Contractor Selection for Public Sustainable Buildings." J. Manag. Eng. 31 (5): 1-7.

[23] USGBC. 2019. LEED v 4 for Building Design and Construction, p. 161.

[24] Donghwan, G., Yong, K. H., and Hyoungsub, K. 2015. "LEED, Its Efficacy in Regional Context: Finding a Relationship between Regional Measurements and Urban Temperature." Energy Build. 86: 687-91.

[25] Lu, Y., Wu, Z., Chang, R., and Li, Y. 2017. "Building Information Modeling (BIM) for Green Buildings: A Critical Review and Future Directions." Autom. Constr. 83 (August): 134-48.

[26] Spatari, S., Eboli, J., Stadel, A., Mitchell, J., and Ryberg, A. 2011. "Intelligent Sustainable Design: Integration of Carbon Accounting and Building Information Modeling." J. Prof. Issues Eng. Educ. Pract. 137 (2): 51-4.

[27] ISO. 2013. ISO 16739:2013-Industry Foundation Classes (IFC) for Data Sharing in the Construction and Facility Management Industries.

[28] BSI. 2014. BS 1192-4: 2014 Collaborative Production of
Information Part 4: Fulfilling Employer's Information Exchange Requirements Using COBie-Code of Practice, p. 58.

[29] Tang, S., Shelden, D. R., Eastman, C. M., Pishdad-Bozorgi, P., and Gao, X. 2019. "BIM Assisted Building Automation System Information Exchange Using BACnet and IFC.” Autom. Constr. 110 (December): 103049.

[30] Centre for Digital Built Britain. 2018 "The Gemini Principles.

[31] Goedert, J. D., and Meadati, P. 2008. "Integrating Construction Process Documentation into Building Information Modeling." Journal of Construction Engineering and Management 134 (7): 509-16.

[32] Chen, Y., and Kamara, J. M. 2011. "A Framework for Using Mobile Computing for Information Management on Construction Sites." Autom. Constr. 20 (7): 776-88.

[33] Re Cecconi, F., Tagliabue, L., Ciribini, A., Maltese, S., and Kovacic, I. 2017. "Green BIM-A Lean Methodology to Support Sustainability Assessment Protocols." In Proceedings of 17th International Multidisciplinary Scientific Geoconference, 889-96. 